\title{
Planetary Nebulae as a Probe of the Local Group Galaxies Evolution
}

\author{
A. Y. Kniazev ${ }^{1}$, S. A. Pustilnik ${ }^{2}$, E. K. Grebel ${ }^{3}$, \\ D. B. Zucker ${ }^{4}$, and P. Vaisanen ${ }^{1}$ \\ ${ }^{1}$ South African Astronomical Obs. Cape Town 7935, South Africa email: akniazev@saao.ac.za \\ ${ }^{2}$ Special Astrophysical Obs. RAS, Nizhnij Arkhyz, 369167, Russia email: sap@sao.ru \\ ${ }^{3}$ Astronomical Institute of the University of Basel, Switzerland email: grebel@astro.unibas.ch \\ ${ }^{4}$ Institute of Astronomy, University of Cambridge, United Kingdom email: zucker@ast.cam.ac.uk
}

Abstract. We present the latest results from our study of $\mathrm{PNe}$ and $\mathrm{H}$ II regions in two Local Group dwarf irregular galaxies IC 10 and NGC 6822.

Keywords. galaxy evolution, galaxy abundances

\section{Introduction}

The nearest galaxies are an excellent laboratory for studies of galaxy evolution. Their star formation histories can be obtained with the color-magnitude diagram of resolved stars. However, the results are model-dependent and should be compared with other, additional observational data that can be obtained for these galaxies. One such complementary means is a study of Planetary Nebulae (PNe) that can be used simultaneously as age, kinematics, metallicity tracers and as secondary distance indicators. Additionally, the abundances from both HII regions and PNe allow one to derive an approximate enrichment history for a galaxy from intermediate ages to the present day and permit a measurement of the abundances at different locations.

\section{PNe in IC10 and NGC6822}

We observed two PNe candidates in IC10 with 6m SAO RAS telescope and have found that the brightest candidate is not a genuine PN but a close pair of compact HII regions. We have found that another observed $\mathrm{PN}$ candidate is a true PN, and thus is the first confirmed PN in IC 10. We give an estimate of the age of PN's progenitor of $\sim 450 \mathrm{Myr}$, which relates its formation to the period during the strongest SF episode in IC 10 within the last 5 Gyr.

Five PNe candidates in the Local Group dwarf irregular galaxy NGC 6822 were observed with the recently commissioned Robert Stobie Spectrograph on SALT (Southern African Large Telescope) in long-slit mode. All observed PNe candidates are true PNe. We measured the electron temperature, the electron density, and element abundances for $\mathrm{He}, \mathrm{N}, \mathrm{O}$, and $\mathrm{Ne}$ or $\mathrm{S}$. Our preliminary calculated average $\mathrm{O} / \mathrm{H}$ for these $\mathrm{PNe}$ is consistent with the average value of $12+\log (\mathrm{O} / \mathrm{H})=8.11 \pm 0.10$, measured for HII regions.

\section{Acknowledgements}

S. A. P. would like to acknowledge the support from IAU (grant No.12330) and Czech Academy of Sciences. 